\title{
Effect of gamma linolenic acid pretreatment on diabetic neuropathy in rats
}

\author{
Nishikant $^{1}{ }^{*}$, Janardhan Singh $^{1}$, Sushma Sood $^{2}$, Vijayshanker $^{3}$
}

${ }^{1}$ Department of Pharmacology,
${ }^{2}$ Department of Physiology,
${ }^{3}$ Department of Biochemistry,
Pt. B.D. Sharma PGIMS,
Rohtak 124001, Haryana, India

Received: 10 April 2013

Revised: 12 April 2013

Accepted: 14 April 2013

\section{*Correspondence to:}

Dr. Nishikant,

Email: drnks77@gmail.com

(C) 2013 Nishikant et al. This is an open-access article distributed under the terms of the Creative Commons

Attribution License, which permits unrestricted use, distribution, and reproduction in any medium, provided the original work is properly cited.

\begin{abstract}
Background: The present study was planned to evaluate the preventive effects of gamma linolenic acid (GLA) on STZ induced diabetic neuropathy

Methods: Streptozotocin (STZ) induced diabetic neuropathy in rats was monitored by measuring blood sugar levels, body weight, motor nerve conduction velocity (MNCV) and nociception. Forty rats were divided into 4 groups of 10 each. Group 1: control (vehicle), Group 2: STZ (50 mg/kg, i.v., single injection), Group 3: Gamma linolenic acid (50 mg/ kg, p.o., daily + STZ), Group 4: STZ + Insulin (4 units/kg, s.c., bid). Similar protocol was used for other parameters also.
\end{abstract}

Results: Gamma linolenic acid pretreatment failed to reduce blood sugar levels in diabetic rats but prevented deterioration of motor nerve conduction velocity as compared to STZ diabetic rats. A significant weight gain was observed in STZ diabetic rats pretreated with GLA as compared to rats received STZ alone. Hyperalgesia induced by STZ was antagonized by GLA

Conclusions: Thus gamma linolenic acid prevents the development of neuropathic changes induced by STZ in rats.

Keywords: Diabetic neuropathy, Gamma linolenic acid, STZ, MNCV, Hyperalgesia

\section{INTRODUCTION}

Diabetes remains the most common cause of nontraumatic limb loss in the world with diabetic peripheral neuropathy the most prominent culpable factor. ${ }^{1}$ Diabetic neuropathy (DN) is characterized by altered excitability which can lead to either "positive" (paresthesia, dysesthesia, pain) or "negative" symptoms (hypesthesia, anesthesia). ${ }^{2}$ Pathologically DN is characterized by progressive degeneration as well as impaired regenerative ability of peripheral nerve fibers, resulting in progressive loss and dying back of the longest nerve fibers innervating the distal limb. These changes are associated with progressive impairment of nerve function leading to impaired sensitivity in the limb which sometimes is associated with troublesome pain. ${ }^{3}$
The abnormalities underlying DN appears to be multiple and involve metabolic, neuronal and vascular mediated defects. Hyperglycemia induced enhancement of polyol pathway flux has been suggested to reduce nerve myoinositol levels and phosphoinositide turnover, compromising $\mathrm{Na}^{+}-\mathrm{K}^{+}$ATPase activity. The resultant changes in ionic homeostasis, microangiopathy resulting in ischemia and lack of neurotropic factors may be responsible for a number of functional and morphological defects including eventual axonopathy. ${ }^{4,5}$ The extent of reversal of neuropathic damage with metabolic control in overt neuropathy varies widely and is unpredictable with published reports of both impressive therapeutic success and failure. ${ }^{6}$ Reactive oxygen species provide reduction in nerve blood flow and endoneurial hypoxia in rat sciatic nerve which may be sufficiently severe so as to 
contribute to dysfunction. Thus free radical injury and oxidative stress have been implicated in pathogenesis of diabetes. $^{7}$

To date irrespective of the pathogenesis there is no satisfactory treatment of DN other than attempting to decrease the symptoms such as paraesthesia, pain, muscle weakness and postural hypotension. ${ }^{2}$ Glycemic control may slow the progression of DN. Aldose reductase inhibitors have been disappointing in the treatment of human DN. ${ }^{8}$ Recently beneficial role of gamma linolenic acid (GLA) has been suggested in DN. ${ }^{9}$

Gamma linolenic acid (GLA) is an omega - 6 polyunsaturated fatty acid which exist in high concentrations in cell membranes as structural phospholipids are essential to cell integrity and viability. ${ }^{10}$ GLA and linolenic acid are essential components of myelin and neuronal cell membrane. ${ }^{11}$ Evening primrose (Openothera Biennis) has attracted attention as a medicinal plant, since the oil of the seed contain high concentration of GLA. GLA has been shown to exert beneficial effects in rheumatoid arthritis, ${ }^{12}$ atopic eczema, ${ }^{13}$ acute respiratory distress syndrome, ${ }^{14}$ Asthma, ${ }^{15}$ ulcerative colitis, ${ }^{16}$ cancer, ${ }^{17}$ and osteoporosis. $^{18}$ Investigations have demonstrated that GLA treatment improved autoimmune diseases ${ }^{12}$ and diabetic neuropathy via an anti inflammatory mechanism. ${ }^{19}$ GLA has shown promising results in the treatment of diabetic complications. ${ }^{20}$

GLA forms dihomo- gamma linolenic acid (DGLA) which can be converted to prostaglandin $\mathrm{E}_{1}\left(\mathrm{PGE}_{1}\right)$ or to arachidonic acid by product. $\mathrm{PGE}_{1}$ has anti inflammatory, antiplatelet and vasodilatory properties. ${ }^{11}$ In patients with type I and type II diabetes decreased production of $\mathrm{PGE}_{1}$ has been reported. ${ }^{21}$ Therefore, supplementation with products rich in linolenic is likely to beneficial in delaying the progression of DN. Therefore, present study was undertaken to evaluate role of gamma linolenic acid in STZ induced diabetic neuropathy in rats.

\section{METHODS}

Albino rats of either sex weighing 200-250 g were maintained under standard conditions with food and water ad libitum. Streptozotocin was purchased from Sigma Inc. (USA). Gamma linolenic acid (GLA 120) was purchased from Dr. Reddy's Laboratories Ltd., Hyderabad (India).

Induction of diabetes: Rats were made diabetic with a single intravenous injection of streptozotocin $(50 \mathrm{mg} / \mathrm{kg})$ in $0.05 \mathrm{~mol} / \mathrm{L}$ citrate buffer, $\mathrm{pH} 4.5$. Samples for blood sugar were obtained after $48 \mathrm{hrs}$ of STZ and every 2 weeks thereafter. Blood glucose levels were determined by the glucose oxidase method. ${ }^{22}$ Rats showing serum glucose levels greater than $250 \mathrm{mg} / \mathrm{dl}$ after $48 \mathrm{hrs}$ of STZ were considered diabetic.
Body weight: Body weight $(\mathrm{g})$ of all the animals were recorded initially (0 week) and at weeks 4, 6, 8, 10 and 12.

Electrophysiological studies: Electromyograph (Medicor, Budapest) was used to measure motor nerve conduction velocity (MNCV). ${ }^{23}$ Rats were anaesthetized with ether to give light anesthesia and ensure minimum interference with nerve conduction. To minimize the effect of differences in body temperature on MNCV, animals were placed under a $40 \mathrm{~W}$ light bulb. Left leg of rats was shaved and cleaned. MNCV was measured in the sciatic- tibial conducting motor system at weeks $0,4,8$, 12. The left sciatic nerve was stimulated with square pulses $(2 \mathrm{HZ})$ at the sciatic notch and tibial nerve at the ankle by superamaximal (8V) stimulation. Responses were recorded from small muscles of the foot by surface electrode. MNCV was calculated by subtracting distal from proximal latency (millisecond) and difference was divided by the distance (millimeter) between the two stimulating electrodes giving the MNCV in meter/second.

MNCV $(\mathrm{M} / \mathrm{S})=$ Distance between two points of stimulation (in $\mathrm{mm}$ )/Latency (from proximal distal point) to onset of muscle action potential

Nociceptive test: Nociceptive response was evaluated using the tail flick test to thermal stimulation by an analgesiometer. ${ }^{24}$ The heat intensity was adjusted so that the rats had control (pre drug) tail flick latencies of 4 to 5 seconds. A cut off latency of $10 \mathrm{sec}$ was used to prevent damage to the tail. Tail withdrawn from heat source was taken as end point. The initial (control) reaction time was recorded in all animals and compared with drug treated groups every 2 weeks for 12 weeks.

\section{Experimental design}

Rats were divided in to 4 groups of 10 each. Group 1 served as control (vehicle). Group 2 were given STZ (50 $\mathrm{mg} / \mathrm{kg}$, i.v.) single injection. Group 3 received gamma linolenic acid $(50 \mathrm{mg} / \mathrm{kg}$, p.o., daily by gavage) 5 days prior to STZ injection and continued for 12 weeks. Group 4 were injected STZ + regular insulin (4 units/ kg, s.c., twice daily for 12 weeks to make rats euglycemic.

\section{Statistical analysis}

Results were statistically analyzed by comparing with controls using student's ' $t$ ' test (unpaired). Probability values less than 0.05 were considered significant. Values were expressed as mean $\pm \mathrm{SEM}$.

\section{RESULTS}

Blood sugar: Blood sugar level in control group was mean $90.2 \pm 1.23$ and remained almost same at weeks 4 , $6,8,10$ and 12. A significant $(\mathrm{p}<0.001)$ and sustained 
increase in blood sugar levels was observed in animals injected STZ. Gamma linolenic acid $(50 \mathrm{mg} / \mathrm{kg}$, p.o., daily) pretreatment failed to alter blood sugar levels in STZ diabetic rats. Insulin treatment prevented the development of STZ induced hyperglycemia in rats. Results are shown in table 1.

Table 1: Effects of GLA (50 mg/kg, p.o., daily) pretreatment on blood sugar levels in diabetic rats.

\begin{tabular}{|lllllll|}
\hline Groups & \multicolumn{2}{c|}{ Blood sugar (mg/dl) } \\
\hline & 0 week & 4 weeks & 6 weeks & 8 weeks & 10 weeks & 12 weeks \\
\hline 1 (Control) & $90.2 \pm 1.23$ & $91.4 \pm 1.40$ & $91.9 \pm 0.83$ & $91.7 \pm 1.22$ & $92.2 \pm 1.35$ & $91.3 \pm 0.91$ \\
\hline 2 (STZ) & $92.4 \pm 1.09$ & $262.3 \pm 1.96^{*}$ & $267.8 \pm 2.08^{*}$ & $263.3 \pm 1.96^{*}$ & $266.9 \pm 2.29^{*}$ & $271.7 \pm 2.14^{*}$ \\
\hline 3 (GLA+STZ) & $90.8 \pm 1.07$ & $245.3 \pm 1.83^{*}$ & $247.9 \pm 1.96^{*}$ & $250.7 \pm 2.14^{*}$ & $240.5 \pm 3.16^{*}$ & $232.0 \pm 4.16^{*}$ \\
\hline 4 (Insulin+ STZ) & $91.4 \pm 1.40$ & $101.2 \pm 1.64$ & $101.8 \pm 1.74$ & $101.0 \pm 1.71$ & $95.5 \pm 1.29$ & $96.5 \pm 0.84$ \\
\hline
\end{tabular}

$* \mathrm{p}<0.001$ when compared with control (student's 't' test).

Body weight: Rats from control group (1) showed a gradual increase in body weight with time during the study period. On the other hand, there was gradual reduction in body weight in animals treated with STZ. Pre treatment with gamma linolenic acid (GLA; 50 $\mathrm{mg} / \mathrm{kg}$, p.o., daily) prevented the reduction in body weight in diabetic animals. The interesting finding of the study was that GLA pretreated animals showed body weight even higher than animals of control group. Insulin treatment partly prevented the reduction in body weight in diabetic animals. Results are shown in figure 1.

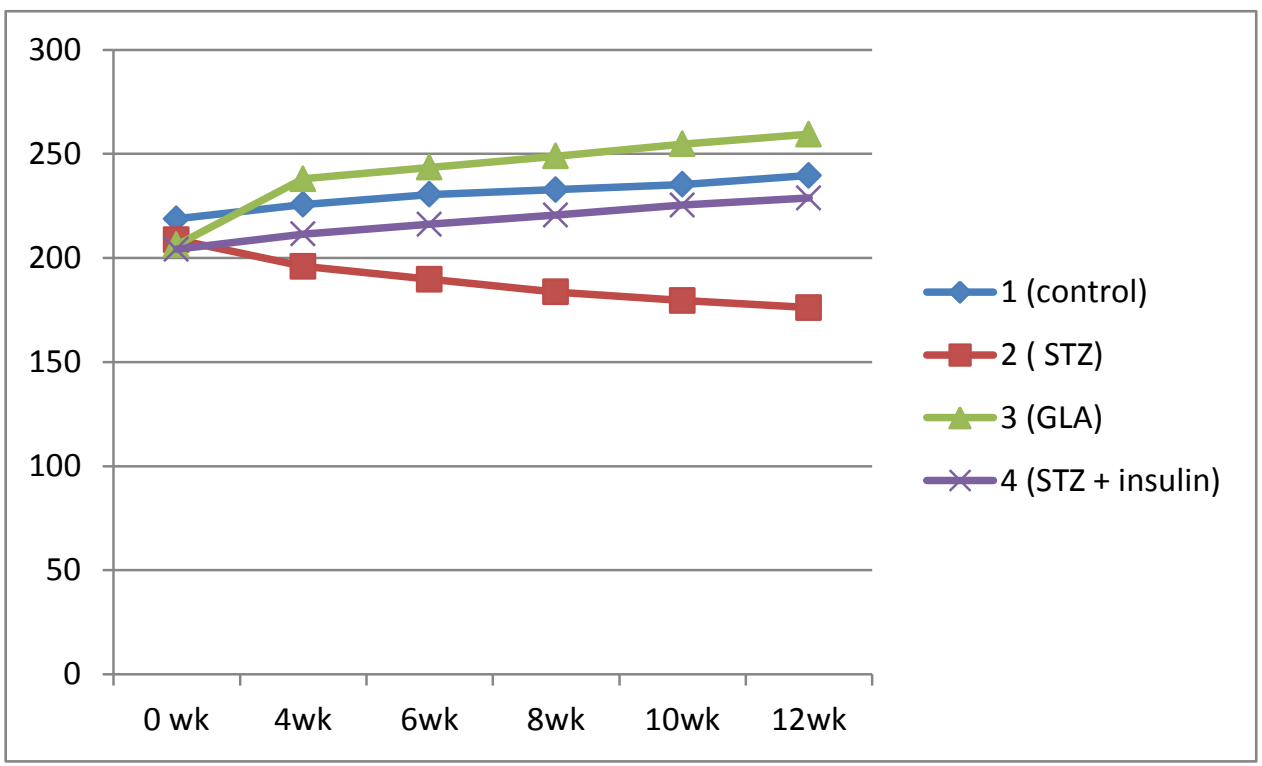

Figure 1: Effect of GLA $(50 \mathrm{mg} / \mathrm{kg}$, p.o., daily) pretreatment on body weight (g) in STZ diabetic rats.

MNCV: As shown in figure 2, age matched control rats showed statistically non significant increase in MNCV recorded at 4,8 , and 12 weeks as compared with initial value (0 week). STZ diabetic rats showed a gradual decrease in MNCV. The MNCV was not significantly different from the initial value up to week 4 . However, 8 weeks onwards the decrease in MNCV was highly significant $(<0.01)$ compared with initial value. Gamma linolenic acid $(50 \mathrm{mg} / \mathrm{kg}$, p.o., daily) pretreatment resulted in an increase in MNCV compared with diabetic animals. The increase at weeks 8 and 12 was highly significant compared with animals receiving STZ alone but remained less compared with control. Insulin pretreatment prevented the reduction in MNCV in diabetic rats. The increase in MNCV was significant compared with diabetic rats but remained lower than that of control group. 


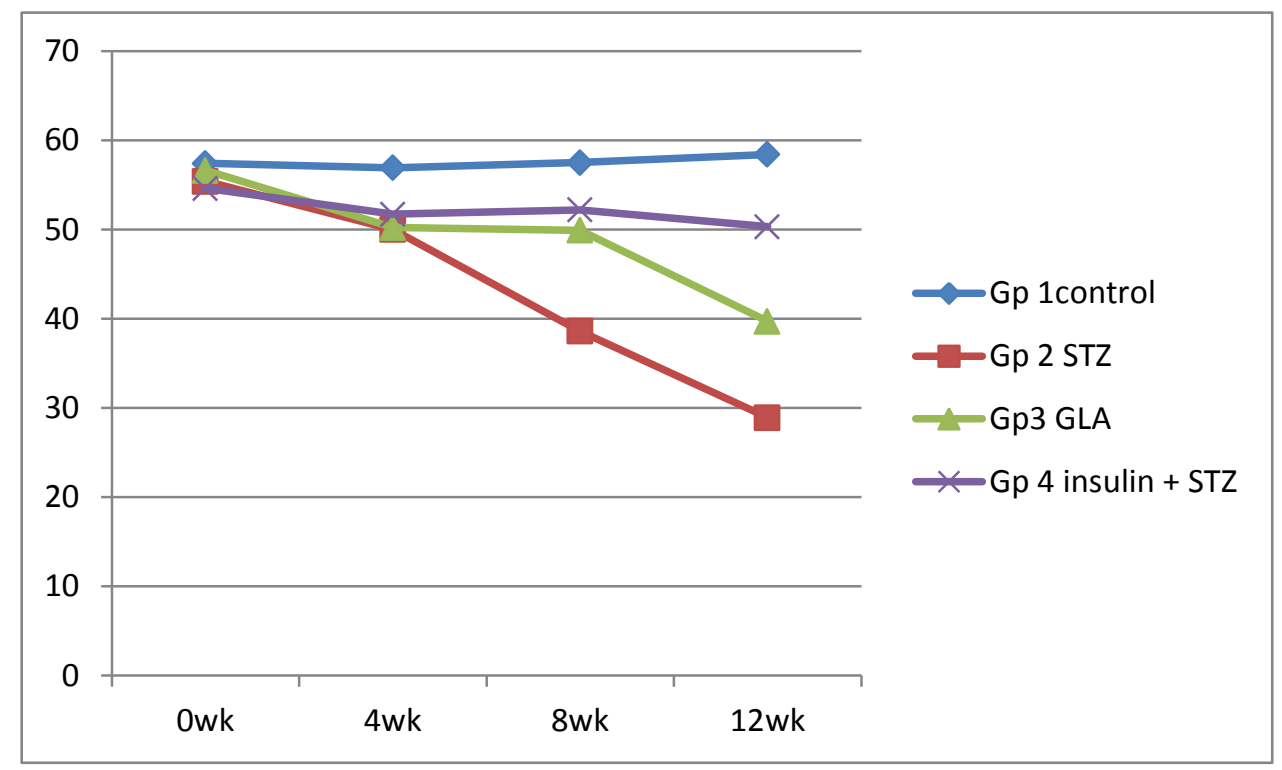

Figure 2: Effect of GLA (50 mg/kg, p.o., daily) and insulin pretreatment on motor nerve conduction velocity in rats with STZ induced diabetic neuropathy.

Table 2: Effect of gamma linolenic acid (GLA) pretreatment on tail flick reaction time to the thermal stimulation in rats with STZ induced diabetic neuropathy. Data represented as mean \pm SEM.

\begin{tabular}{|lllllll|}
\hline Groups & \multicolumn{5}{c|}{ Tail flick reaction time (seconds) } \\
\hline & 0 week & 4 weeks & 6 weeks & 8 weeks & 10 weeks & 12 weeks \\
\hline 1 (Control) & $4.60 \pm 0.24$ & $4.70 \pm 0.21$ & $4.90 \pm 0.17$ & $4.80 \pm 0.20$ & $4.40 \pm 0.16$ & $4.70 \pm 0.21$ \\
\hline 2 (STZ) & $4.80 \pm 0.20$ & $4.60 \pm 0.16$ & $4.00 \pm 0.21$ & $3.40 \pm 0.16^{*}$ & $3.20 \pm 0.13^{*}$ & $2.60 \pm 0.16^{*}$ \\
\hline 3 ( GLA+STZ) & $4.70 \pm 0.21$ & $4.50 \pm 0.16$ & $4.40 \pm 0.16$ & $4.20 \pm 0.13^{\#}$ & $3.90 \pm 0.17^{\#}$ & $3.50 \pm 0.16^{\#}$ \\
\hline 4 ( Insulin+STZ) & $4.86 \pm 0.15$ & $4.70 \pm 0.21$ & $4.60 \pm 0.24$ & $4.40 \pm 0.16^{\#}$ & $4.56 \pm 0.03^{\#}$ & $4.12 \pm 0.15^{\#}$ \\
\hline
\end{tabular}

$* \mathrm{p}<0.01$ when compared with control, \# $\mathrm{p}<0.05$ when compared with STZ (student's 't' test).

Nociception: As shown in table 2, administration of STZ resulted in hyperalgesic response in rats, observed only 8 weeks onward. GLA pretreatment antagonized the STZ induced hyperalgesia in diabetic rats. The increase in reaction time was significant $(\mathrm{p}<0.05)$ at weeks 8,10 , and 12 compared with STZ diabetic animals, though it was less when compared with control group. Tail flick reaction time in insulin + STZ treated animals was not significantly different when compared with control group. However, there was significant difference in reaction time when compared with animals receiving only STZ i.e. diabetic control.

\section{DISCUSSION}

Results from the present study revealed marked hyperglycemia reduced MNCV and hyperalgesia after hyperglycemia in rats. STZ acts through a common pathway dependent on the formation of single strand breaks in $\beta$ cell DNA. These breaks than activate nuclear enzymes poly (ADP - ribose) synthase in $\beta$ cell to such an extent that the stores of its substrate nicotinamide adenine dinucleotide (NAD) become critically depleted. ${ }^{25}$
There is selective destruction of pancreatic $\beta$ cells. In addition, there is increased catabolism in diabetes leading activation of glycogenolysis, gluconeogenesis, proteolysis, lipolysis, and ketogenesis responsible for hyperglycemia and weight loss.

Hyperalgesia observed did not follow the acute changes in the blood glucose levels. The blood glucose level was elevated as early as 3 days after STZ injection, where as no change in nociception threshold was present at this point of time. Significant hyperalgesia was observed after only 8 weeks of STZ administration. These results indicate that acute hyperglycemia is not responsible for hyperalgesia. However chronic hyperglycemia may lead to some unknown secondary metabolic, functional or structural changes in diabetic nerve. It is clear that gamma linolenic acid (GLA) does not act by improving blood glucose levels, as it failed to reduce the hyperglycemic effect of STZ in rats.

Antinociceptive effect of GLA observed in STZ diabetic rats may be due to its neuroprotective, vasodilatory and antioxidant properties. Metabolites of GLA include 
essential fatty acid (EFA) derivatives such as DGLA and AA which are important constituents of phospholipids of neuronal membranes and also prostaglandin $\mathrm{E}_{1}$ which can be formed from DGLA. ${ }^{10}$ GLA is likely to have desirable effect both on the neuronal phospholipids, structure and the micro circulation. A recent study has revealed that diabetes alters myelin's phospholipid, fatty acid and cholesterol content in pattern that can modify membrane fluidity. ${ }^{26}$ It has been suggested that regeneration capacity of nerve fibers in STZ diabetes is impaired. ${ }^{9}$ Evidence point to increased oxidative stress in diabetic neuropathy either due to increased oxidative stress in diabetic neuropathy due to increased production of reactive oxygen species (ROS) or defective scavenging of free radical. $^{7}$ The protective effect of GLA in nerve fibers may be explained by their powerful action of free radical scavenging activity, by stimulating growth factor and promoting nerve fiber regeneration. ${ }^{9}$ Treatment with antioxidants in STZ diabetic rats was shown to have protective effect on $\beta$ cell apoptosis. ${ }^{27}$ Therefore antioxidant for free radical scavenging effect of GLA may be beneficial in reducing neural damage in diabetic nerves.

Several studies have shown pivotal role of micro vascular abnormalities in the pathogenesis of diabetic neuropathy. Decreased nerve blood flow and endoneurial hypoxia in sciatic nerve of rat which is sufficiently severe so as to contribute nerve dysfunction. ${ }^{5}$ Hypoxia per se can induce severe changes and slowing of nerve conduction velocity in peripheral nerves. ${ }^{3}$ GLA is a precursor of vasodilatory prostaglandin $\mathrm{E}_{1}$. GLA via conversion into prostaglandin $\mathrm{E}_{1}$, exhibits vasodilatory effects on microneurial blood flow and hence oxygen dependent metabolic process leading to better nerve function. Hence, vasodilatation could be an important factor in mediating beneficial effect of GLA in STZ induced DN. GLA therefore may improve blood flow and oxygen tension in the diabetic nerve.

In addition marked weight gain was observed in diabetic rats treated with GLA strongly suggested anabolic effect of this compound or GLA may affect significantly metabolism (protein, fat, nitrogen) except carbohydrate metabolism. Insulin pretreatment almost completely prevented the development of STZ induced diabetic complication in rats. The preventive role of insulin was explained on the basis of its metabolic effects. Therefore, finding of the study demonstrate preventive effect of GLA on STZ induced neuropathy in rats. Certainly the drug affects other metabolic / anabolic functions including energy metabolism and antagonizes STZ induced catabolic processes.

\section{Funding: No funding sources}

Conflict of interest: None declared

Ethical approval: The study was approved by the Institutional Animal Ethical Committee

\section{REFERENCES}

1. Zimmy S, Sehatz H, Pfohl M. The role of limited joint mobility in diabetic patients with an at risk foot. Diabetes Care 2004;27:942-6.

2. Thomas PK, Tomlison DR. Diabetic and hypoglycemic neuropathy in Dyck PJ, Thomas PK, Griffin JW, Low PA, Pdulo JF (editors): Peripheral neuropathy. Philadelphia, Saunnders; 1993:1219-50.

3. Sima AA, Greene DA. Diabetic neuropathy in elderly. Drugs Aging 1995;6:125-35.

4. McCarty MF. Nitric oxide deficiency, leukocyte activation and resultant ischemia area critical to the pathogenesis of diabetic retinopathy/ neuropathy preventive potential of antioxidants, essential fatty acids, chromium, ginkgolides and pentoxifylline. Med Hypotheses 1998;50:435-49.

5. Vinik AI, Erbas T, Sansberry KB. Small fiber neuropathy and neurovascular disturbances in diabetes mellitus. Exp Clin Endocrinol Diabetes 2001;109(suppl.2):s451-73.

6. Mancuso JM. Impact of health literacy and patient trust on glycemic control in an urban population. Nurs Health Sci 2010;12:94-104.

7. Lee NY, Kang YS. Regulation of taurine transport at the blood placental barrier by calcium ion, PKC activator and oxidative stress conditions. J Biomed Sci 2010;17(suppl1):s37.

8. Nicolucci A, Crici F, Cavaliere D. A meta-analysis of trials of aldose reductase inhibitor in diabetic peripheral neuropathy. Diabet Med 1996;13:1017-26.

9. Omran OM. Histopathological study of evening primrose oil effects on experimental diabetic neuropathy. Ultrastruct Pathol 2012;36:222-7.

10. Benatti P, Peluso G, Nicolai R, Calvani M. Polysaturated fatty acids: biochemical, nutritional and epigastric properties. J Am Cell Nutr 2004;23:281-302.

11. Carter JP. Gamma linolenic acid as a nutrient. Food Tech 1998;42:72-82.

12. Zurier RB, Rosseti RA, Jacobson EW, De Marco DM, Liu NY, Tenming JE, et al. Gamma linolenic acid treatment of rheumatoid arthritis. A randomized, placebo controlled trial. Arthritis Rheum 1996;39:1808-17.

13. Andereassi M, Forleo P, Di- Lorio A. Efficacy of gamma linolenic acid in the treatment of patients with atopic dermatitis. J Int Med Res 1997;25:26674.

14. Pacht ER, DeMichele SJ, Nelson JL. Enteral nutrition with eicosopentaenoic acid, gamma linolenic acid and antioxidants reduces alveolar inflammatory mediators and protein influx in patients with acute respiratory distress syndrome. Crit Care Med 2003;31:491-500.

15. Surette ME, Koomenis IL, Edens MB. Inhibition of leucotrine biosynthesis by a novel dietary fatty acid formulation in patients with ectopic asthma: a randomized, placebo controlled, parallel group, prospective trial. Clin Ther 2003;25:972-9. 
16. Middleton SJ, Naylor S, Wodner J, Hunter JO. A double blind randomized placebo controlled trial of essential fatty acid supplementation in the maintenance of remission of ulcerative colitis. Aliment Pharmacol Ther 2002;16:1131-50.

17. Kenny FS, Gee JM, Nichalson RI. Effect of dietary GLA + tamoxifen on the growth, ER expression and fatty acid profile of ER positive breast cancer xenograft. Int J Cancer 2001;92:342-7.

18. Kruger MC, Coetzer H, de winter R, et al. Calcium, gamma linolenic acid and eicosapentanoic acid supplementation in senile osteoporosis. Aging (Milano) 1998;10:385-94.

19. Keen H, Payan J, Allawi J, Walker J, Jamal GA, Weir AI. Treatment of diabetic neuropathy with gamma linolenic acid. The gamma linolenic acid multicenter trial group. Diabetes Care 1993;16:8-15.

20. Suresh Y, Das UN. Long chain polyunsaturated fatty acids and chemically induced diabetes mellitus. Effects of omega- 6 fatty acids. Nutrition 2003;19:93-114.

21. Brenner RR. Nutritional and hormonal factors influencing desaturation of essential fatty acids. Prog Lipid Res 1982;20:41-7.
22. Trinder P. Enzymatic determination of blood glucose. Ann Clin Biochem 1969;6:24-8.

23. DE Koning P, Gispen WH. Org.2766 improves functional and electrophysiological aspects of regenerating sciatic nerve in the rat. Peptides 1987;8:415-22.

24. Davies OL, Ranventos J, Walpole AL. Methods for evaluation of analgesic activity using rats. $\mathrm{Br} \mathrm{J}$ Pharmacol Chemother 1946;1:255-64.

25. Musabayane CT, Tufts MA, Mapanga RF. Synergistic antihyperglycemic effects between plantderived oleanolic acid and insulin in streptozotocininduced diabetic rats. Ren Fail 2010;32:832-9.

26. Cermenati G, Affiati F, Cermenati S. Diabetes induced myelin abnormalities are associated with an altered lipid pattern: protective effects of LXR activation. J Lipid Res 2012;53:300-10.

27. Srinivasan A, Menon VP, Pariaswamy V, Rajsekaran KN. Protection of pancreatic beta-cell by the potential antioxidant bis-o-hydroxycinnamoyl methane, analogue of natural curcuminoid in experimental diabetes. J Pharm Pharm Sci 2003;6:327-33.

doi:10.5455/2319-2003.ijbcp20130617

Cite this article as: Nishikant, Singh J, Sood S

Vijayshanker. Effect of gamma linolenic acid pretreatment on diabetic neuropathy in rats. Int $\mathbf{J}$ Basic Clin Pharmacol 2013;2:320-5. 